\title{
Process Neural Network Method: Case Study I: Discrimination of Sweet Red Peppers Prepared by Different Methods
}

\author{
Sevcan Unluturk, ${ }^{1}$ Mehmet S. Unluturk, ${ }^{2}$ Fikret Pazir, ${ }^{3}$ and Alper Kuscu ${ }^{4}$ \\ ${ }^{1}$ Food Engineering Department, Izmir Institute of Technology, 35430 Izmir, Turkey \\ ${ }^{2}$ Department of Software Engineering, Izmir University of Economics, Sakarya Caddesi No. 156 Balcova, 35330 Izmir, Turkey \\ ${ }^{3}$ Food Engineering Department, Ege University, 35040 Izmir, Turkey \\ ${ }^{4}$ Faculty of Agriculture, Suleyman Demirel University, 32260 Isparta, Turkey
}

Correspondence should be addressed to Mehmet S. Unluturk, suleyman.unluturk@ieu.edu.tr

Received 2 November 2010; Accepted 3 February 2011

Academic Editor: Enrico Capobianco

Copyright (๑) 2011 Sevcan Unluturk et al. This is an open access article distributed under the Creative Commons Attribution License, which permits unrestricted use, distribution, and reproduction in any medium, provided the original work is properly cited.

\begin{abstract}
This study utilized a feed-forward neural network model along with computer vision techniques to discriminate sweet red pepper products prepared by different methods such as freezing and pureeing. The differences among the fresh, frozen and pureed samples are investigated by studying their bio-crystallogram images. The dissimilarity in visually analyzed bio-crystallogram images are defined as the distribution of crystals on the circular glass underlay and the thin or the thick structure of crystal needles. However, the visual description and definition of bio-crystallogram images has major disadvantages. A methodology called process neural network (ProcNN) has been studied to overcome these shortcomings.
\end{abstract}

\section{Introduction}

Excluding analytical chemistry approaches for the evaluation of food quality, there are numerous alternative methods (holistic methods) that do not focus on the analysis of single substances but regard foodstuffs as a whole. This approach is based on the proposition that life itself is more than the sum of its single constituent parts. The organization and structure of food are important factors. The conservation of this organization and structure is a sign of high food quality, and structural decomposition is synonymous with plant death. The purpose of complementary analysis methods is to help characterize the vital activity [1]. The biocrystallization method is one of the most important complementary analysis methods.

Pfeiffer [2] originally introduced the term biocrystallization, which is also called "sensitive crystallization" and "copper chloride crystallization". Engqvist [3] later initiated the biocrystallization method. This method was developed from the viewpoint that living organisms do not just exist as substances, but have directing and organizing "structuring forces" $[4,5]$. These structuring forces direct the form and function of the organism [6]. The method is based on the crystallographic phenomenon that when adding specific ionic substances, or in general all organic substances, to an aqueous solution of dehydrate $\mathrm{CuCl}_{2}$, biocrystallograms with reproducible dendritic structures are formed during crystallization. Crystallograms that are produced on the basis of pure $\mathrm{CuCl}_{2}$ exhibit a merely peripheral distribution of crystals on the circular glass underlay, with a diameter of $90 \mathrm{~mm}$ [4]. In contrast, biocrystallograms produced on the basis of biological substances, such as plant extracts (fresh sweet red pepper samples in this study), exhibit crystal structures covering the entire glass underlay (Figure 1).

The biocrystallograms that are produced from agricultural products, such as vegetables, grains, fruits, and milk samples are based on three components: (a) an aqueous solution or extract of the sample in question, (b) an aqueous solution of dehydrate copper chloride, and (c) purified water. Any kind of additive will change the copper chloride 


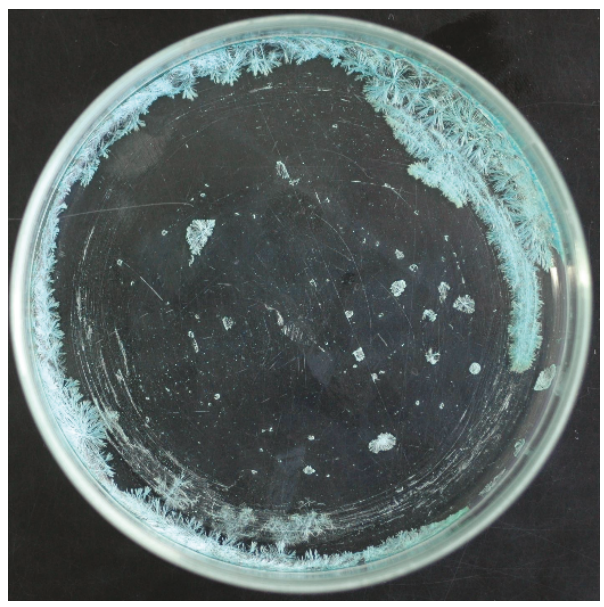

(a)

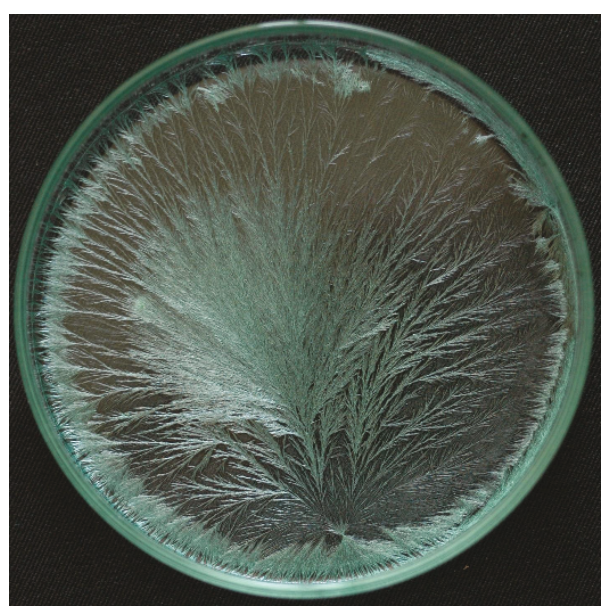

(b)

FIGURE 1: (a) Crystallogram obtained from basis of aqueous $\mathrm{CuCl}_{2} \cdot 2 \mathrm{H}_{2} \mathrm{O}$ (blank). (b) Biocrystallogram obtained from conventionally grown fresh sweet red pepper.

crystallization. The process is influenced by the qualitative and quantitative variations in the macromolecules of the biological extracts, thus allowing food quality assessment [5]. When used to study human blood, the results are correlated with groups of pathologies and the concentration of blood protein, and are predictive of worsening illnesses. Early diagnosis seems possible in cancer research or in occupational medicine (risk indicators) [7].

The phenomena of biocrystallograms are based on ramification patterns, which may be divided into three major stages, extending from the center in all directions to the periphery of the image (Figure 2). In the first or 1-zone biocrystallogram, when increasing concentrations of agricultural extracts are applied relative to a given fixed concentration of $\mathrm{CuCl}_{2}$, transparent needles with enormous star-like formations extend in all directions to the periphery. The second, 2-zone structure goes through the crystallization center when it is divided by a vertical and horizontal axis. The needles are pointed, predominantly transparent, and of relatively equal length in the middle zone. These morphological features may be described by means of plant morphological terms, such as stems, branches, and needles. The last stage of a biocrystallogram is an optimal degree divided into a 3-zone structure with a center zone, middle zone, and marginal zone. In the third stage, a biocrystallogram exhibits various macro and microscopic morphological features that reflect the quality of the sample in question [5].

The biocrystallization method comprises two main parts (Figure 3). The first is pattern formation, which starts in a laboratory and continues until the crystallogram picture is completely generated in the chamber. The second part is pattern recognition. This is where the evaluation tools will be responsible for perceiving and differentiating between images [5].

There are two tools currently used to evaluate an image visual evaluation and computerized image analysis. In visual evaluation the images in question are evaluated based on the judgment of a trained human using discrete reference scales

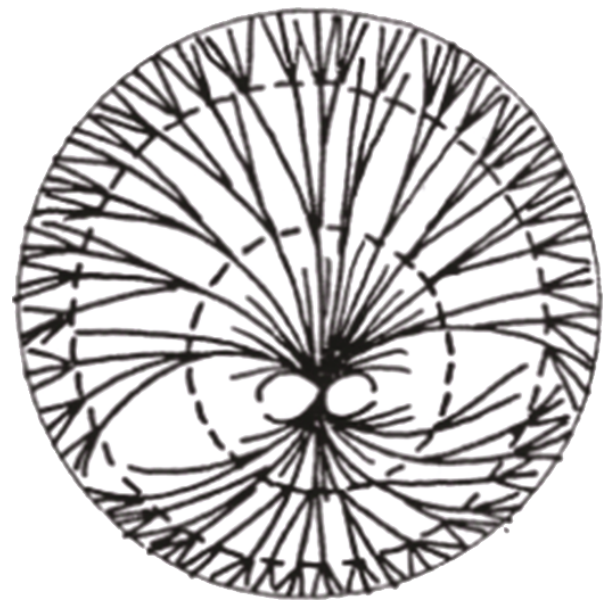

Figure 2: Biocrystallization method based on a phenomenon of dendritic pattern formation during crystallization from an aqueous solution containing plant extracts and $\mathrm{CuCl}_{2}[2]$.

arranged in connection with picture phenomena. Computerized image analysis interprets the image by using the fundamental knowledge of texture analysis. Such techniques have been explored and applied with the biocrystallization method [5].

Computerized image analysis techniques may meet the demand for such methods. Ideally, an image analysis procedure should reflect all of the characteristics of a biocrystallogram as a three-dimensional, colored ramification structure, coordinated with zones relative to the center. However, due to the present limitations set by computational capacity and speed, simpler approaches are preferable. In a limited number of previous studies, encouraging results have been reported [4].

Computerized image analysis tools increase the objectivity of the method and allow the analysis of large numbers of crystallization images [8]. This paper presents a unique neural network model, called process neural network (ProcNN), 


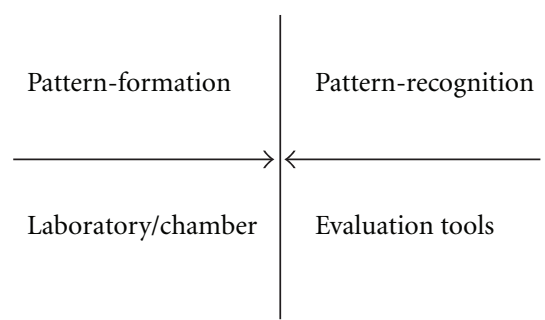

Figure 3: The detail of biocrystallization method [8].

along with computer vision techniques as an effective analysis tool. The applicability of this tool is tested for discrimination of sweet red peppers (Capsicum annuum L.) prepared by different methods such as freezing and pureeing. As an effective computer vision technique, RGB (red, green, blue) space is used since sweet red pepper fruit digital images are captured and saved in RGB (red, green, blue) space. For each component in RGB space, we performed two calculations; the mean and standard deviation. The mean characterizes the average color properties of sweet red pepper, while standard deviation provides a measure of color variation. Since three digital values assigned to every pixel of sweet red pepper color image, 6 color features were extracted for RGB color space, including the mean and the standard deviation of each color component [9].

\section{Sweet Red Pepper Extract and Copper Chloride Concentrations}

Conventionally grown sweet red pepper fruits (Capsicum annuиm L.) were obtained from Ege University Agricultural Faculty Research, Applied and Production Farm in Menemen (2005-2006; Izmir, Turkey). Fresh sweet red peppers were quickly transferred to the laboratory. They were sorted, cleaned, washed, dried, packaged under vacuum and frozen at $-25^{\circ} \mathrm{C}$. They were stored at the same temperature until used. The preparation steps of pureed samples include removal of the stem and kernel parts of fresh peppers, grating, thermal blanching at $95-100^{\circ} \mathrm{C}$ for 10 minutes, hot filling and sterilization at $115^{\circ} \mathrm{C}$ for 20 minutes. Sterilized pureed samples were refrigerated at $4^{\circ} \mathrm{C}$ until used.

In order to prepare sample extracts from fresh, frozen, and sweet red peppers, the large peppers were initially chopped into small pieces and passed through a kitchen type blender (Braun MR 404, Hesse, Germany). The homogenate was first passed through a cheese cloth to remove debris particles and then filtered. The fruit juice was diluted to $1 \%$ with tridistilled water. $\mathrm{CuCl}_{2} \cdot 2 \mathrm{H}_{2} \mathrm{O}$ solution was also prepared at a $16 \%$ concentration with tridistilled water.

The optimal mixing ratio for the sample extract and copper chloride influences the crystallization pattern. Therefore, the optimum sample and $\mathrm{CuCl}_{2} \cdot 2 \mathrm{H}_{2} \mathrm{O}$ concentrations were determined to be $1 \%$ and $16 \%$, respectively.

For each biocrystallogram, a $1 \mathrm{~mL}$ sample ( $1 \%$ concentration) was mixed with $3 \mathrm{~mL} \mathrm{CuCl} 2 \cdot 2 \mathrm{H}_{2} \mathrm{O}$ (16\% concentration) per plate at chamber conditions of $25^{\circ} \mathrm{C}$ and $55 \%$ relative humidity $[11,12]$. The copper chloride was crystallized after 16-18 hours. A total of 840 biocrystallograms were produced from sweet red pepper fruit in 2005 and 2006. Only single centered biocrystallograms were evaluated, the others (multicentered) were discarded.

2.1. Image Acquisition and Capture. Images were captured using a digital camera model DMC-FZ5 (5 Mp) (Panasonic, NJ, USA). The camera was positioned vertically over the sample at a certain distance. The angle between the camera lens, the lighting source, and ambient illumination were fixed and kept the same for all the sample pictures. After that, the images were transferred and stored in a PC as a JPEG format of "high resolution" and "superfine quality".

2.2. Image Processing. All the algorithms for preprocessing of full images and image segmentation were written in MATLAB 6.5 (The Math Works, Inc., MA, USA). MATLAB code for pre-processing and image segmentation of a single full image is given below:

$$
\begin{aligned}
& s=\left[{ }^{\prime} \text { 07.09.2006 taze Kristal } 0^{\prime}\right. \text { int2str(iStart)'.jpg']; } \\
& I=\operatorname{imread}(s) \text {; } \\
& D=i m 2 \text { double }(I) \text {; } \\
& r=D(:,:, 1) \text {; } \\
& g=D(:,:, 2) \text {; } \\
& b=D(:,:, 3) \text {; } \\
& r=r(501: 1100,351: 1250) \text {; } \\
& g=g(501: 1100,351: 1250) \text {; } \\
& b=b(501: 1100,351: 1250) \text {; } \\
& d=\text { get_coeffs } 2(r, g, b) \text {. }
\end{aligned}
$$

Inside get_coeffs $2(r, g, b)$ function, the $r, g, b$ values are used to calculate the mean and the standard variation for red, green, and blue components. Returned vector has six values which correspond to RGB scale image. They are used as input to the feed-forward neural network.

2.3. Image Segmentation. Each $1488 \times 2240$ pixel RGB images was cropped to $600 \times 900$ pixel size $(r=r(501: 1100$, $351: 1250) ; g=g(501: 1100,351: 1250) ; b=b(501: 1100,351$ : 1250)); where $r, g, b$ is the red, green, and blue components). These images were then used in the decision process, as the aim of these software-based procedures is to eventually replace the human visual decision-making process.

\section{Process Neural Network Architecture}

Figure 4 shows a $1488 \times 2240$ RGB color biocrystallogram images of fresh, pureed, and frozen sweet red peppers. In these images, each pixel has three components corresponding to Red, Green, and Blue. We reduced the image size to 600 $\times 900$ (Figure 5), and then we calculated the mean and standard deviation of Red, Green, and Blue components of these images. 


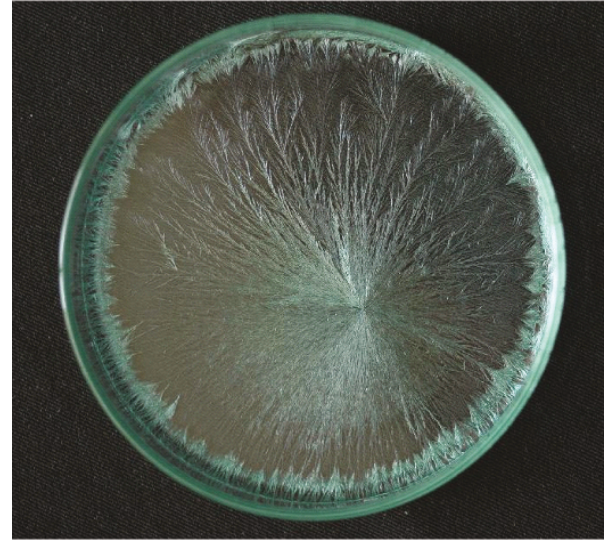

(a)

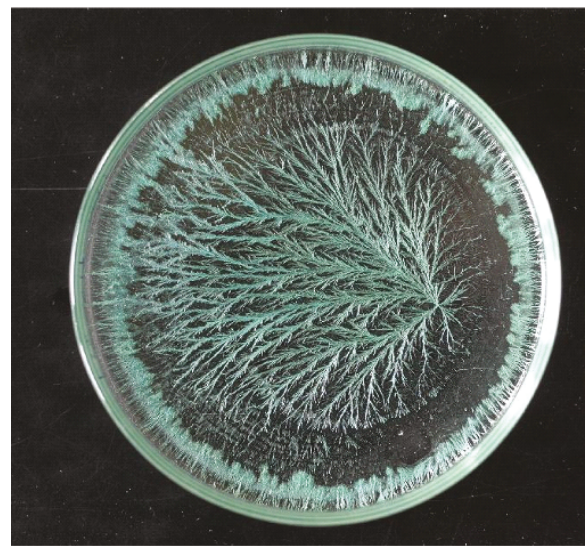

(b)

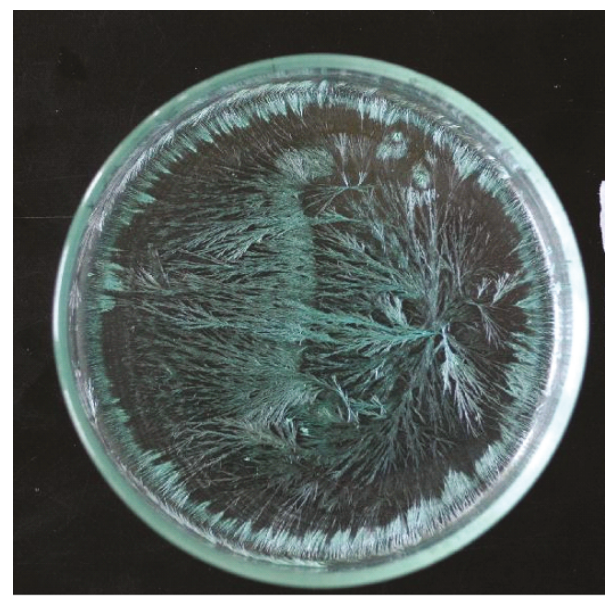

(c)

FIGURE 4: Biocrystallogram images of (a) fresh, (b) pureed, and (c) frozen sweet red peppers [10].

As a result, we extracted 6 features for each image. These features are the input to the feed-forward neural network. There is one output node only whose value is -0.9 if the features belong to one type of class such as fresh sweet red pepper fruit, or else 0.9 if the features belong to the other type of class such as pureed sweet red pepper fruit. We chose 7 as the number of hidden neurons. During the training phase, if the learning does not get better, we will increment the number of hidden neurons by two every other 400 epochs [13]. For the training algorithm, we used back-propagation algorithm [14-16]. Table 1 shows the training statistics for the ProcNNs.

Epoch is defined as the presentation of the entire training set to the ProcNN, and sum-squared error is defined as a measure of how well ProcNN is doing at a particular point during its training [14-16]. For example, it took only 44 epochs to train the neural network for classification of fresh and pureed samples. In the training phase, all the samples (100\%) were correctly classified by each neural network. Sum squared error chosen for these neural networks was 0.009 . Figure 6 shows a fully interconnected feed-forward neural network. It has six inputs, 7 hidden neurons and one output neuron.
We examined the output statistics of the training phase and decided to choose 0 as the decision factor (see Section 6). The best performance that we obtained was from the ProcNN for fresh and pureed samples. Testing output for all 70 fresh samples was less than zero and for all 70 frozen samples, it was bigger than zero. We reached $100 \%$ recognition.

There is also an alternative neural network approach for the same problem. We can apply Bayes optimal decision rule since we have two classes to separate [14]. Following section discusses this method.

\section{Bayes Optimal Decision Rule}

Classes are defined as:

$$
\begin{aligned}
& H_{0}=\text { Fresh, } \\
& H_{1}=\text { Processed (pureed). }
\end{aligned}
$$

The prior probability of an unknown image being drawn from class $k$ is ${ }^{h} k(k=0,1)$. The cost of making a wrong decision for class $k$ is ${ }^{v} k$. Note that the prior probability ${ }^{h} k$ and the cost probability ${ }^{v} k$ are taken as being equal, 


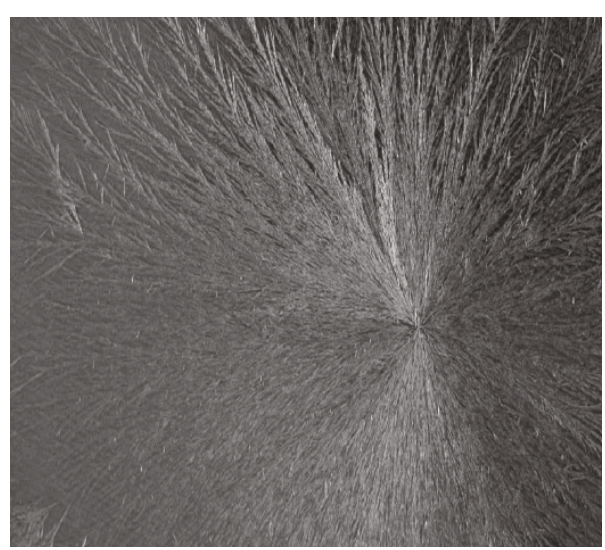

(a)

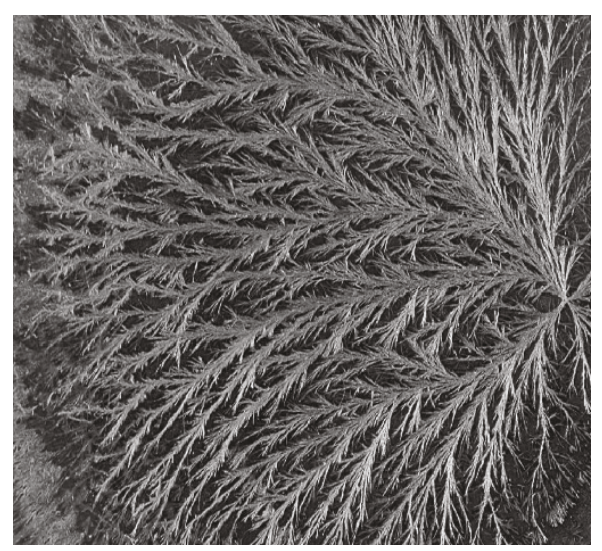

(b)

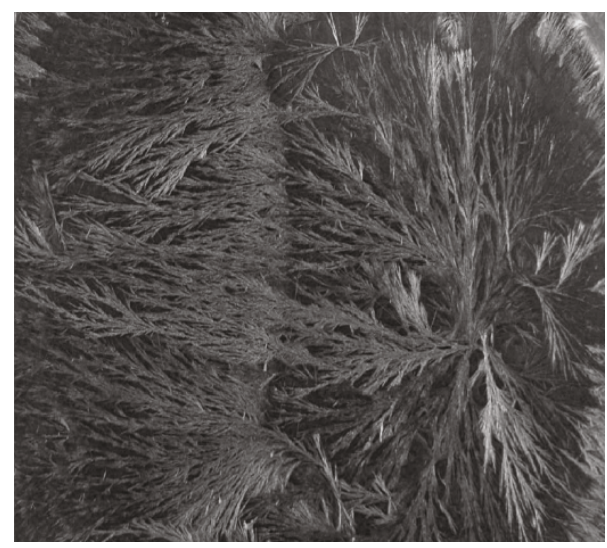

(c)

FIgURE 5: Reduced image size $(600 \times 900$ pixel $)$ for (a) fresh, (b) pureed, and (c) frozen sweet red peppers.

TABle 1: Training statistics for ProcNNs.

\begin{tabular}{lccc}
\hline Neural network type & \# of Epochs & Sum-squared error & Recognition \\
\hline ProcNN (fresh and pureed) & 44 & 0.009 & $100 \%$ \\
ProcNN (fresh and frozen) & 70 & 0.009 & $100 \%$ \\
ProcNN (frozen and pureed) & 29 & 0.009 & $100 \%$ \\
\hline
\end{tabular}

and hence can be ignored. The problem is to find a neural network model for determining the class from which an unknown image is taken. If we know the probability density functions $f_{k}(\vec{X})$ for all classes, the Bayes optimal decision rule [14] can be used to classify $\vec{X}$ into class $k$ if

$$
h_{k} \vartheta_{k} f_{k}(\vec{X})>h_{m} \vartheta_{m} f_{m}(\vec{X})
$$

where $k \neq m$.

A major problem with (1) is that the probability density functions of the classes are unknown. We can use Gram-Charlier series expansion to estimate these unknown probability density functions. In this case, the training set for the neural network consists of Gram-Charlier coefficients. Our objective in this study was to use these coefficients for classification. If the neural network is trained with known Gram-Charlier coefficients, then to determine the class of an unknown image, we need only to feed its Gram-Charlier coefficients. The next section describes the Gram-Charlier series expansion.

\section{Gram-Charlier Series}

The Gram-Charlier series expansion of the probability density function of a random variable with mean $\mu$ and variance $\sigma^{2}$ can be represented as

$$
\rho(x)=\frac{1}{\sigma} \sum_{i=0}^{\infty} c_{i} \phi^{(i)}\left(\frac{x-\mu}{\sigma}\right),
$$

where $\phi(x)$ is a Gaussian probability density function and $\phi^{(i)}(x)$ represents the $i$ th derivative of $\phi(x)$. For normalized 


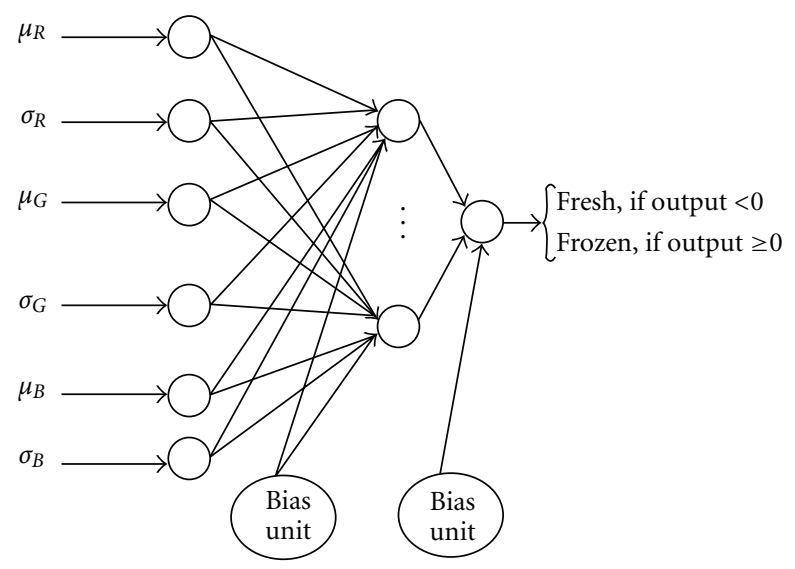

Figure 6: Process neural network (ProcNN) for fresh and pureed samples.

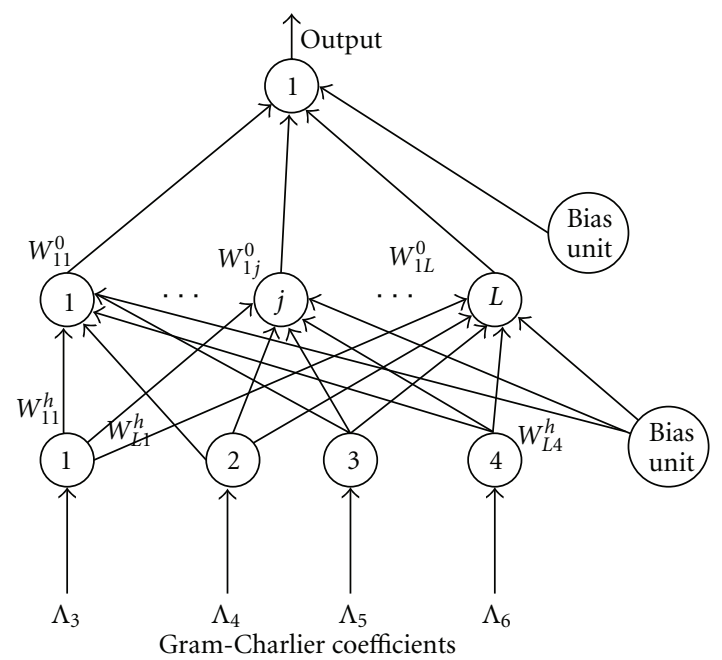

FIGURE 7: Back propagation neural network. If output $\geq 0$, then input type of biocrystallogram sample image belongs to process class (pureed) or it belongs to fresh class (where $L$ is 19 ).

data where $\left(\mu=0, \sigma^{2}=1, c_{0}=1\right)$, the above equation can be simplified to

$$
\begin{gathered}
\rho(x)=\left(\phi(x)+c_{3} \phi^{(3)}(x)+c_{4} \phi^{(4)}(x)+c_{5} \phi^{(5)}(x)\right. \\
\left.+c_{6} \phi^{(6)}(x)+\cdots\right),
\end{gathered}
$$

where $c_{i}$ coefficients are related to the central moments of $\phi(x)$. In a sense, derivatives of the Gaussian function in (3) provide us with the class type information for the sweet red pepper. Furthermore, $c_{i} \phi^{(i)}$ are orthogonal functions that present unique information about the process type sweet red pepper class distribution. This leads us to conclude that the procNN based on the decomposition of the probability density function by the Gram-Charlier series is well suited for fresh/processed (pureed) pepper class discrimination. Let's define $\beta(x)$ as

$$
\beta(x)=\frac{1}{\sqrt{2 \pi}} e^{\left((-1 / 2) x^{2}\right)} .
$$

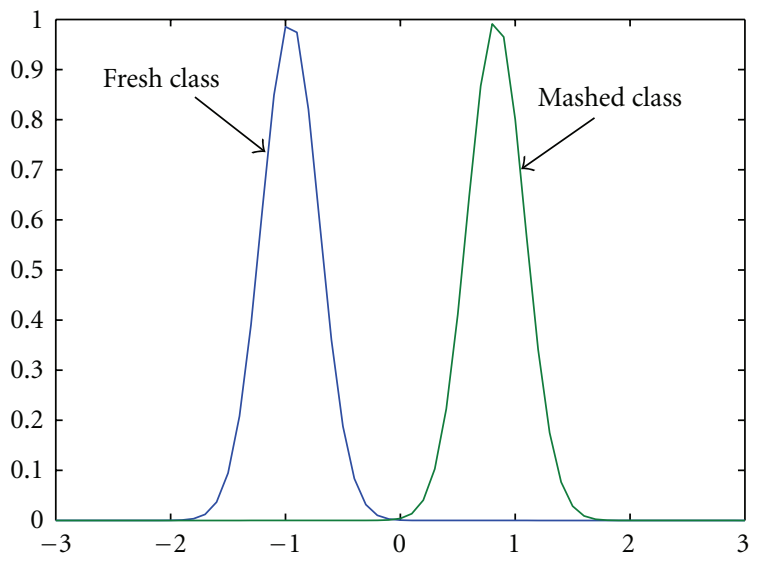

(a)

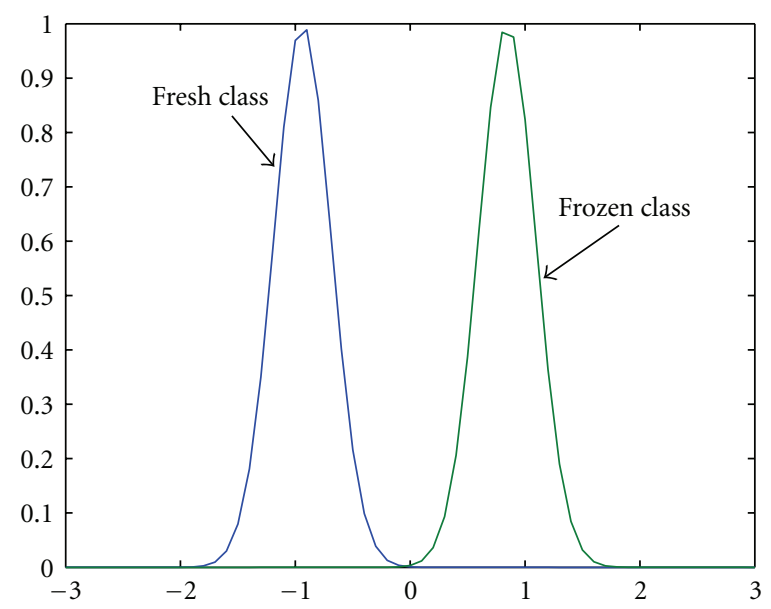

(b)

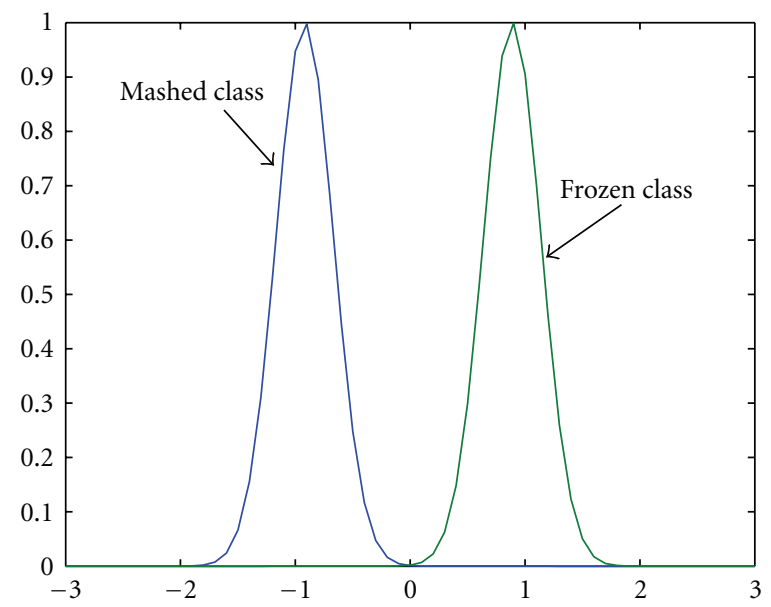

(c)

Figure 8: Output density functions for training phase of (a) freshpureed (shown as mashed) classes, (b) fresh-frozen classes, (c) pureed (shown as mashed)-frozen classes. 
Then, the probability density function becomes

$$
\rho(x)=\beta(x)\left\{1+\frac{1}{6} \Lambda_{3} A_{3}+\frac{1}{24}\left(\Lambda_{4}-3\right) A_{4}+\cdots\right\}
$$

where

$$
\begin{aligned}
& A_{i}(x)= x^{i}-\frac{i^{[2]}}{2 \cdot 1 !} x^{i-2}+\frac{i^{[4]}}{2^{2} \cdot 2 !} x^{i-4}-\frac{i^{[6]}}{2^{3} \cdot 3 !} x^{i-6}+\cdots, \\
& i^{[k]}=\left(\begin{array}{l}
i \\
k
\end{array}\right)=\frac{i !}{(i-k) !}, \\
& m_{k}=\frac{1}{n} \sum_{i=1}^{n}[x(i)]^{k}, \\
& \Lambda_{3}=-\frac{m_{3}-3 m_{2} m_{1}+2 m_{1}^{3}}{3 !}, \\
& \Lambda_{4}=\frac{m_{4}-4 m_{3} m_{1}+6 m_{2} m_{1}^{2}-3 m_{1}^{4}}{4 !} \\
& \Lambda_{5}=-\frac{m_{5}-5 m_{4} m_{1}+10 m_{3} m_{1}^{3}-10 m_{2} m_{1}^{3}+4 m_{1}^{5}}{5 !} \\
& \Lambda_{6}= \frac{m_{6}-6 m_{5} m_{1}+15 m_{4} m_{1}^{2}}{6 !} \\
&-\frac{20 m_{3} m_{1}^{3}+15 m_{2} m_{1}^{4}-5 m_{1}^{6}}{6 !} .
\end{aligned}
$$

Equation (6) is the so-called Gram-Charlier series [17] and the polynomial $A_{i}(x)$ is called the Tchebycheff-Hermite polynomial.

The training matrix was prepared using the GramCharlier coefficients. Each column in the training matrix represented one set of these Gram-Charlier coefficients with a length of 4 . Note that the amount of columns in the training set could be any number. The bigger the number, the better the discrimination achieved by the neural network for the target biocrystallogram red pepper single centered images.

The results obtained when testing this neural network show that biocrystallograms of sweet red pepper targets can be detected with $56 \%$ accuracy. The number of hidden neurons for the back propagation neural network was 19. It took 2500 epochs for this neural network to train to reach a sum-squared error of 0.009 . In testing, if the output of the output $\geq 0$, then the input Gram-Charlier coefficients belonged to class $H_{1}=$ processed (pureed). If the output of the output $<0$, then the input Gram-Charlier coefficients belonged to class $H_{0}=$ fresh (Figure 7 ).

Comparing ProcNN and Gram-Charlier neural network, we can decide that representing images with Gram-Charlier coefficients cause to lose the color information which is important to detect the process types of the sweet red pepper. On the other hand, ProcNN uses the mean and the color variation of color components which helps the ProcNN reach the performance between $85 \%$ and $100 \%$.

\section{Results}

We created three ProcNNs. One ProcNN is used to classify fresh and pureed, the second one is used to classify fresh and frozen, and the last one is used to classify frozen and pureed samples. The $1488 \times 2240$ pixel biocrystallogram images were acquired in a lab and cropped to $600 \times 900$ pixel images depicting either a fresh, pureed, or frozen sweet red pepper. Within these images, a set of 140 images was utilized to train each process neural network. Half of this set belonged to one type of pepper class and the other half of the set belonged to the other type of pepper class. A new set of 140 images was then prepared to test each ProcNN performance in a similar way.

Figure 8 shows the output training statistics for fresh and pureed, fresh and frozen, and pureed and frozen sweet pepper samples. We chose 0 as the decision factor (Figure 8(a)). During testing this ProcNN for discrimination of fresh and pureed samples, any output whose value is greater and equal to 0 , we decide the sample belongs to pureed class; otherwise it belongs to fresh class. Testing output for all 70 fresh samples was less than zero, and for all 70 frozen samples, testing output was bigger than zero. We reached $100 \%$ recognition.

We also chose 0 as the decision factor for ProcNN in the fresh and frozen samples (Figure 8(b)). During testing this ProcNN, any output whose value is greater and equal to 0 , we decide the sample belongs to frozen class; otherwise it belongs to Fresh class. Testing output for 63 out of 70 fresh samples was less than zero, and for 65 out of 70 frozen samples, testing output was bigger than zero. We reached $92 \%$ recognition.

For discrimination of fresh and frozen samples, we also chose 0 as the decision factor for this procNN (Figure 8(c)). During testing this ProcNN, any output whose value is greater and equal to 0 , we decide the sample belongs to fresh class; otherwise it belongs to frozen class. Testing output for 57 out of 70 fresh samples was less than zero, and for 62 out of 70 frozen samples, testing output was bigger than zero. We reached $85 \%$ recognition.

This high level of recognition suggests that ProcNN methodology and color measurements obtained from RGB color space is a promising method for the discrimination of sweet red pepper products prepared by different methods using its biocrystallogram images.

\section{Conclusion}

In this study, we developed a neural network to determine whether sweet red peppers are processed or not. We use biocrystallogram images of sweet red peppers for this neural network model since these images taken from a lab bear information related to the process type of pepper. However, this information is not readily quantifiable and lacks uniquely recognizable features. Therefore, a neural network becomes appealing for classifying these images, because neural network is trainable. The optimal values for the neural network weights were estimated using the back propagation algorithm. Experimental measurements of the 
pepper were utilized to train and test the process neural network. This network showed a remarkable 100\% classification performance. Parallel classification performance was also achieved when training the neural network. These results are encouraging and suggest that neural networks are potentially useful for discriminating sweet red peppers processed by different methods. Furthermore, the process neural network renders practical advantages such as realtime processing, adaptability, and training capability. It is important to point out that similar neural network designs can be used in classification of food grains' images, detection of contaminated food products, evaluating the surface quality of food raw materials, determination of quality features of foods, such as object recognition, geometrical parameters, surface colour, and in other areas such as medical ultrasonic imaging for tissue characterization and diagnosis, industrial defect discrimination, and so forth.

\section{References}

[1] W. Harms, "Quality of organic food," Genetic Engineering Newsletter-Special Issue, vol. 16, pp. 1-11, 2004.

[2] E. Pfeiffer, Studium von Formkräften an Kristallizationen, Naturwissenschaftliche Sektion am Goetheanum, Dornach, Switzerland, 1931.

[3] M. Engqvist, Gestaltkrafte des Lebendigen, Klostermann, Frankfurt am Main, Germany, 1970.

[4] J. O. Andersen, C. B. Henriksen, J. Laursen, and A. A. Nielsen, "Computerised image analysis of biocrystallograms originating from agricultural products," Computers and Electronics in Agriculture, vol. 22, no. 1, pp. 51-69, 1999.

[5] A. Meelursarn, Statistical evaluation of texture analysis from the biocrystallization method: effect of image parameters to differentiate samples from different farming systems, Doctoral dissertation, University of Kassel, Department of Organic Food Quality and Food Culture, Witzenhausen, Germany, December 2006.

[6] J. Bloksma, M. Northolt, and M. Huber, Parameters for Apple Quality Part-1, Louis Bolk Institute, 2001.

[7] J. G. Barth, "Cristallisation avec additif, cas particulier du chlorure cuivrique et de ses applications," Phytothérapie, vol. 2, no. 6, pp. 183-190, 2004.

[8] P. Doesburg and M. Huber, "Biocrystallisation and Steigbild results at the Louis Bolk Instituut," Elemente Der Naturwissenschaft, no. 87, pp. 118-123, 2007.

[9] C. Zheng, D.-W. Sun, and L. Zheng, "Correlating colour to moisture content of large cooked beef joints by computer vision," Journal of Food Engineering, vol. 77, no. 4, pp. 858863,2006

[10] A. Kuscu, Organik ve Konvansiyonel Kirmızıbiber ve Ürünlerinin Ayırt Edilebilme Yöntemleri ve Kalite Özelliklerinin Incelenmesi, Ph.D. thesis, Ege Üniversitesi Fen Bilimleri Enstitüsü, Izmir, Turkey, 2008.

[11] U. R. Balzer-Graf, U. Köpke, and U. Geier, "Research on Quality in Organic Agriculture by Picture Forming Methods, Understanding the Quality of Organic Horticultural Products (Short Course on)," Izmir, Turkey, May 2001.

[12] M. Szulc, F. Cordeiro, A. Maquet, and E. Anklam, "Application of a multivariate design approach for maximisation of the observed differences between organically and conventionally grown wheat grains in biocrystallisation method," in Proceedings of the 1st Scientific FQH Conference, p. 78, Fibl, Switzerland, November 2005.

[13] M. Islam, A. Sattar, F. Amin, X. Yao, and K. Murase, "A new adaptive merging and growing algorithm for designing artificial neural networks," IEEE Transactions on Systems, Man, and Cybernetics B, vol. 39, no. 3, pp. 705-722, 2009.

[14] T. Masters, Practical Neural Networks, Academic Press, New York, NY, USA, 1993.

[15] A. Cichocki and R. Unbehauen, Neural Networks for Optimization and Signal Processing, John Wiley \& Sons, New York, NY, USA, 1992.

[16] J. A. Freeman and D. M. Skapura, Neural Networks Algorithms, Applications, and Programming Techniques, Addison-Wesley, 1991.

[17] M. W. Kim and M. Arozullah, "Generalized probabilistic neural network based classifiers," in Proceedings of the International Joint Conference on Neural Networks, vol. 3, pp. 648-653, 1992. 